\title{
Detection of Social Media Exploitation via SMS and Camera
}

https://doi.org/10.3991/ijim.v13i04.10521

Mohamad Adib Azhar( $\left.{ }^{\square}\right)$, Madihah Mohd Saudi, Azuan Ahmad, Azreena Abu Bakar Universiti Sains Islam Malaysia (USIM), Nilai, Malaysia

jbalika@outlook.com

\begin{abstract}
Internet users all over the world are highly exposed to social media exploitation, where they are vulnerable to be targeted by this cyber-attack. Furthermore, excessive use of social media leads to Internet Addiction Disorder (IAD). Fortunately, social media exploitation and IAD can be monitored and controlled closely based on user's mobile phone surveillance features which are camera, SMS, audio, geolocation (GPS) and call log. Hence to overcome these challenges, this paper presents five (5) Application Programming Interfaces (APIs) and four (4) permissions for SMS and camera that are mostly and widely used with the social media applications. These 9 APIs and permissions matched with $2.7 \%$ of the APIs and permissions training dataset that are related with SMS and camera. This experiment was conducted by using hybrid analysis, which inclusive of static analysis and dynamic analysis, with 1926 training dataset from Brunswick. These 9 APIs and permissions, if being misused by the attacker, could lead to privacy concerns of a mobile device. The finding from this paper can be used as a guidance and reference for the formation of new mobile malware detection technique and modeling in future.
\end{abstract}

Keywords-Social media exploitation, API, permission, SMS, camera, mobile malwares, mobile phone surveillance feature.

\section{Introduction}

Human emotion or desire to browse social media via mobile phone to get latest information, communicate with friends and play game, is currently becoming a trend. Unfortunately, excessive use of social media could lead to Internet Addiction Disorder (IAD) and depression. Recently, World Health Organisations (WHO) has declared gaming as one of the International Classification of Diseases (ICD-11) in year 2018. Hence, it is not impossible in future that social media addiction will be categorized as mental disorder due to its implications and impacts to serious depression and lifestyle.

In a smartphone, 5 main surveillance features which are: SMS, camera, call log, geolocation (GPS) and audio could be exploited by the attacker. They can monitor user's movement and steal confidential information via these surveillance features. In earlier day, Short Message System (SMS) is one of the main mechanisms used by many users for communication. Until now, SMS is still being used for communication 
and authentication of online banking. Apart from SMS, camera becomes as an important element in smartphone selection due to our current lifestyle. Picture can be easily disseminated to social media just in a second. Different platforms such as iOS and Android have been implemented in different smartphones and Android has been ranked as the mostly used worldwide. As a result, it is most targeted by the attackers and malwares due to its open-source distribution [1]. Malware is defined as a software that could infect devices without the owner's consent for malicious intention and it can be categorised as virus, worm, Trojan Horse, adware, spyware, botnet or ransomware. So far, mobile botnet posed the most serious impact to the smartphone users. For an example, in August 2017, WireX botnet spreads among users from 100 countries and it has infected advertising software and launched the DDoS attacks. It hides under system processes and has been taken down from Playstore with the help from Akamai, Flashpoint and Oracle Dyn [2]. In an Android smartphone, every application has limited capability to use smartphone resources and it needs to request permission and Application Programming Interface (API) to perform any task. For an example, once a mobile application (app) is being installed, the mobile app will request a permission to use SMS and camera during first execution or during installation. Once user granted this permission, the app has the authority to send related information and request via SMS and camera.

Features such as API and permission are seen as an opportunity for exploitation [3]. Existing works by [4-12] showed the significant of API and permission usage for exploitation and malwares detection. These works used different analysis techniques such as static analysis, dynamic analysis or hybrid analysis. As for work from [12], MalDozer is proposed to detect the malwares in different of IoT devices, with API as the input. Even in 2018, works by [13-17] also applied the API and Permission in their work. The summarization of work in year 2018 can be referred in Table 1. Nonetheless, none of these works focus on social media app exploitation.

Table 1. Summarisation of related existing works

\begin{tabular}{|c|l|l|l|}
\hline Author & Feature & \multicolumn{1}{|c|}{ Description } & \multicolumn{1}{|c|}{ Challenges } \\
\hline$[12]$ & API & $\begin{array}{l}\text { This paper presents about malware } \\
\text { classification. }\end{array}$ & $\begin{array}{l}\text { Performance issue related with } \\
\text { dataset. }\end{array}$ \\
\hline$[13]$ & $\begin{array}{c}\text { API and } \\
\text { permission }\end{array}$ & $\begin{array}{l}\text { This paper presents how can defend } \\
\text { against poisoning attacks from mal- } \\
\text { wares efficiently. }\end{array}$ & $\begin{array}{l}\text { Improvement needed for feature } \\
\text { selection and classifier. }\end{array}$ \\
\hline$[14]$ & $\begin{array}{c}\text { API and } \\
\text { permission }\end{array}$ & $\begin{array}{l}\text { This paper presents malware detection } \\
\text { based on accuracy, recall and F- } \\
\text { measure. }\end{array}$ & $\begin{array}{l}\text { Performance issue related with the } \\
\text { feature selection of the permission } \\
\text { list. }\end{array}$ \\
\hline$[15]$ & $\begin{array}{c}\text { API and } \\
\text { permission }\end{array}$ & $\begin{array}{l}\text { This paper presents model based on } \\
\text { computational processes. }\end{array}$ & $\begin{array}{l}\text { Improvement for limitation of } \\
\text { malware classification based on } \\
\text { binary format. }\end{array}$ \\
\hline$[16]$ & API & $\begin{array}{l}\text { This paper presents malware detection } \\
\text { for anti-virus scanners evasion. }\end{array}$ & $\begin{array}{l}\text { Performance issue related with } \\
\text { training dataset. }\end{array}$ \\
\hline
\end{tabular}

Though each of the existing works has it owns strength, but still lack of discussion on social media exploitation via API and permission. There are five (5) social media 
applications (apps) have been selected for the experiment of our paper. These social media apps are chosen due to the significant impact to the Internet user lifestyle and privacy concerns. Therefore, this paper aims to identify API and permission that are possible to be used for exploitation specifically through SMS and camera.

This paper is organized as follows: Section 2 presents the methodology used in this research, while Section 3 describes the experiments findings carried out in this research and Section 4 includes the summary of the research work.

\section{$2 \quad$ Methodology}

The following Fig. 1 is the illustration of the lab setup for the experiment conducted and Table II displays the software used. Prior matching step of the extracted API and permission, 1926 of dataset from Brunswick have been downloaded for training purpose [18]. 328 of APIs and permissions for mobile botnet have been reverse engineered by using hybrid analysis and being compared with the APIs and permissions extracted from the social media apps. Hybrid analysis is the combination of the static analysis and dynamic analysis. For this experiment the hybrid analysis is being used to ensure the full extraction from the apps are successfully retrieved. Only 1500 dataset from 1926 training dataset are fully functioning for the analysis. As for the testing, 5 social media apps have been selected where their names are being sanitized and displayed as anonymous in this paper to avoid any conflict of interest. These social media apps are among the top 5 in the world with highest usage.

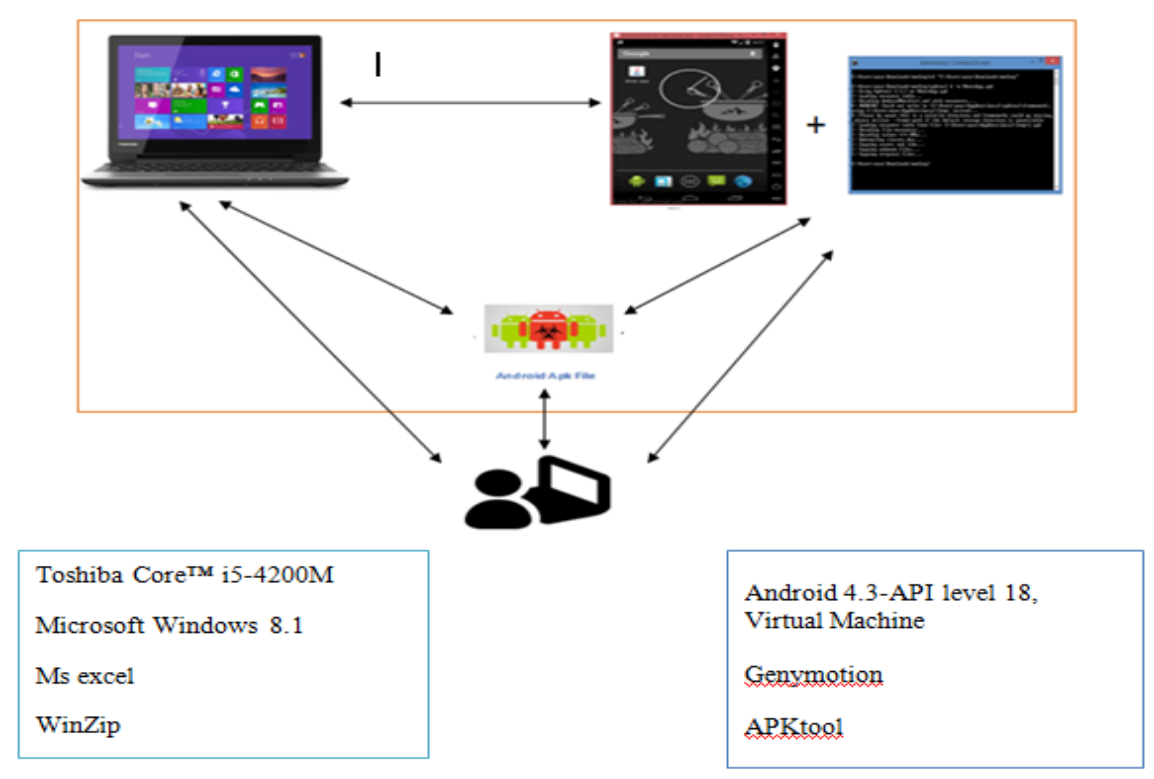

Fig. 1. Lab setup 
Table 2. Software Function

\begin{tabular}{|l|l|}
\hline \multicolumn{1}{|c|}{ Software/Hardware } & \multicolumn{1}{c|}{ Function } \\
\hline Genymotion & It is used as the Android emulator. \\
\hline Show Java Application /APKtool & It is used to decompile APK resource file and extract Permission. \\
\hline Java Decompiler & It is used to extract API. \\
\hline
\end{tabular}

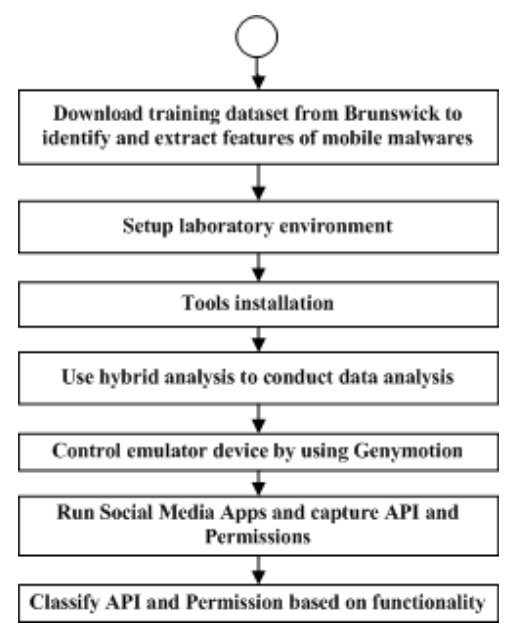

Fig. 2. Overall research processes

Fig. 2 represents the summarization of the whole steps during the experiment. These 5 social media apps of APIs and permissions are being reverse engineered and analyzed, and compared with the existing extracted of 328 mobile botnets APIs and permissions. This is important to classify each of the API and permission as normal or as dataset with an opportunity for malicious exploitation (refer Fig. 3). While Fig. 4, shows an example of permission extraction for the social media app.

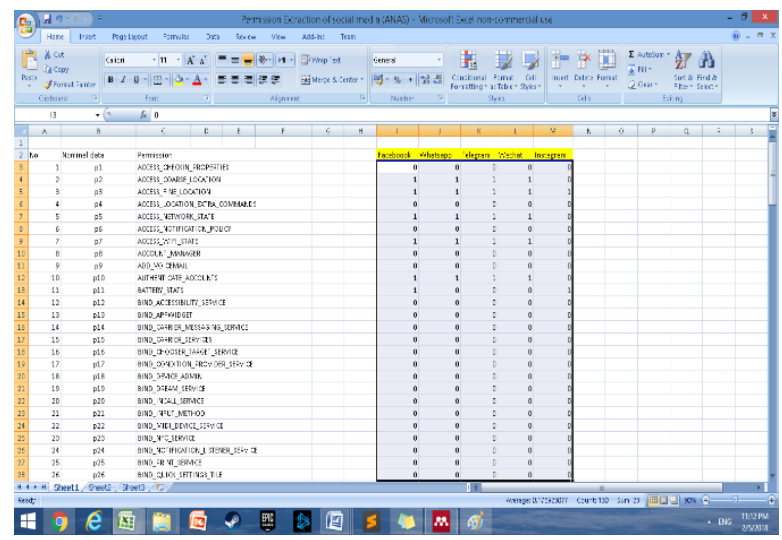

Fig. 3. Comparison between Permissions and APIs with mobile botnet features 


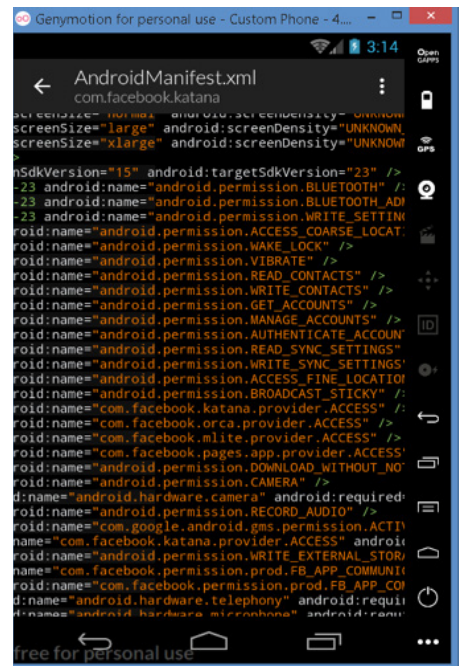

Fig. 4. Example of permission extraction

\section{$3 \quad$ Findings}

The following are the findings of API and permission classification for SMS and camera for mobile botnet from the training dataset and possible exploitation of API and permission in social media apps.

The nominal data in Table III to Table VIII represents the feature representative in symbol. Table III depicts 190 APIs extraction names from the training dataset and Table IV displays 14 APIs that are related with SMS and camera.

Table 3. API extracted from training dataset

\begin{tabular}{|l|l|}
\hline \multicolumn{1}{|c|}{ Nominal data } & \\
\hline AP1 - AP12 $:$ path: android/accounts/AccountManager \\
\hline AP1 & addAccount \\
\hline AP2 & addAccountExplicitly \\
\hline AP3 & blockingGetAuthToken \\
\hline AP4 & getAccounts \\
\hline AP5 & getAccountsByType \\
\hline AP6 & getAuthToken \\
\hline AP7 & getPassword \\
\hline AP8 & invalidateAuthToken \\
\hline API9 & peekAuthToken \\
\hline AP10 & removeAccount \\
\hline AP11 & setAuthToken \\
\hline AP12 & setPassword \\
\hline AP13 $-\boldsymbol{A P 1 8}: \boldsymbol{p a t h}:$ android/app/Activity \\
\hline
\end{tabular}




\begin{tabular}{|c|c|}
\hline AP13 & sendBroadcast \\
\hline AP14 & setContentView \\
\hline AP15 & setPersistent \\
\hline AP16 & startActivity \\
\hline AP17 & startActivityForResult \\
\hline AP18 & startActivityIfNeeded \\
\hline \multicolumn{2}{|c|}{ AP19-AP22 : path: android/app/ActivityManager } \\
\hline AP19 & getRecentTasks \\
\hline AP20 & getRunningTasks \\
\hline AP21 & killBackgroundProcesses \\
\hline AP22 & restartPackage \\
\hline \multicolumn{2}{|c|}{ AP23-AP38 : path: android/app/Activity } \\
\hline AP23 & reportFailedPasswordAttempt \\
\hline AP24 & reportSuccessfulPasswordAttempt \\
\hline AP25 & setActivePasswordState \\
\hline AP26 & AlarmManager;->setTimeZone \\
\hline AP27 & backup/BackupManager;->dataChanged \\
\hline AP28 & Instrumentation;->sendKeyDownUpSync \\
\hline AP29 & KeyguardManager\$KeyguardLock;->disableKeyguard \\
\hline AP30 & KeyguardManager\$KeyguardLock;->reenableKeyguard \\
\hline AP31 & KeyguardManager;-> exitKeyguardSecurely \\
\hline AP32 & NotificationManager;->notify \\
\hline AP33 & Service;->sendBroadcast \\
\hline AP34 & Service;->startActivity \\
\hline AP35 & StatusBarManager;->expand \\
\hline AP36 & WallpaperManager;->setBitmap \\
\hline AP37 & WallpaperManager;->setResource \\
\hline AP38 & WallpaperManager;->suggestDesiredDimensions \\
\hline \multicolumn{2}{|c|}{ AP39 : path: android/appwidget/AppWidgetManager } \\
\hline AP39 & bindAppWidgetId \\
\hline \multicolumn{2}{|c|}{ AP40-AP51 : path: android/bluetooth/BluetoothAdapter } \\
\hline AP40 & cancelDiscovery \\
\hline AP41 & disable \\
\hline AP42 & enable \\
\hline AP43 & getAddress \\
\hline AP44 & getBondedDevices \\
\hline AP45 & getState \\
\hline AP46 & isDiscovering \\
\hline AP47 & isEnabled \\
\hline AP48 & listenUsingRfcommWithServiceRecord \\
\hline AP49 & startDiscovery \\
\hline AP50 & createRfcommSocketToServiceRecord \\
\hline AP51 & getBondState \\
\hline
\end{tabular}




\begin{tabular}{|c|c|}
\hline \multicolumn{2}{|c|}{ AP52-AP54 : path: android/bluetooth/ } \\
\hline AP52 & BluetoothDevice;-> getName \\
\hline AP53 & BluetoothHeadset;->getBatteryUsageHint \\
\hline AP54 & BluetoothSocket;->connect \\
\hline \multicolumn{2}{|c|}{ AP55-AP65 : path: android/content/ContentResolver } \\
\hline AP55 & addPeriodicSync \\
\hline AP56 & getMasterSyncAutomatically \\
\hline AP57 & getSyncAutomatically \\
\hline AP58 & openFileDescriptor \\
\hline AP59 & openInputStream \\
\hline AP60 & openOutputStream \\
\hline AP61 & query \\
\hline AP62 & removePeriodicSync \\
\hline AP63 & setIsSyncable \\
\hline AP64 & setMasterSyncAutomatically \\
\hline AP65 & setSyncAutomatically \\
\hline \multicolumn{2}{|c|}{ AP66-AP74 : path: android/content/Context } \\
\hline AP66 & sendBroadcast \\
\hline AP67 & sendOrderedBroadcast \\
\hline AP68 & sendStickyBroadcast \\
\hline AP69 & setWallpaper \\
\hline AP70 & startActivity \\
\hline AP71 & startService \\
\hline AP72 & ContextWrapper;->sendBroadcast \\
\hline AP73 & ContextWrapper;->setWallpaper \\
\hline AP74 & ContextWrapper;->startActivity \\
\hline \multicolumn{2}{|c|}{ AP75-AP77 : path: android/content/pm } \\
\hline AP75 & PackageManager;->addPreferredActivity \\
\hline AP76 & PackageManager;->clearPackagePreferredActivities \\
\hline AP77 & PackageManager;->setComponentEnabledSetting \\
\hline \multicolumn{2}{|c|}{ AP78 : path: android/ } \\
\hline AP78 & hardware/Camera;->open \\
\hline \multicolumn{2}{|c|}{ AP79-AP88 : path: android/location/LocationManager } \\
\hline AP79 & addGpsStatusListener \\
\hline AP80 & addNmeaListener \\
\hline AP81 & getBestProvider \\
\hline AP82 & getLastKnownLocation \\
\hline AP83 & getProvider \\
\hline AP84 & getProviders \\
\hline AP85 & isProviderEnabled \\
\hline AP86 & requestLocationUpdates \\
\hline AP87 & sendExtraCommand \\
\hline AP88 & setTestProviderEnabled \\
\hline
\end{tabular}




\begin{tabular}{|c|c|}
\hline \multicolumn{2}{|c|}{ AP89-AP95 :path: android/media/AudioManager } \\
\hline AP89 & isBluetoothA2dpOn \\
\hline AP90 & isWiredHeadsetOn \\
\hline AP91 & setBluetoothScoOn \\
\hline AP92 & setMode \\
\hline AP93 & setSpeakerphoneOn \\
\hline AP94 & startBluetoothSco \\
\hline AP95 & stopBluetoothSco \\
\hline \multicolumn{2}{|c|}{ AP96-AP100 : path: android/media/ } \\
\hline AP96 & MediaPlayer;->start \\
\hline AP97 & MediaPlayer;->stop \\
\hline AP98 & MediaRecorder;->setAudioSource \\
\hline AP99 & MediaRecorder;->setVideoSource \\
\hline AP100 & RingtoneManager;->setActualDefaultRingtoneUri \\
\hline \multicolumn{2}{|c|}{ AP101-AP108 : path: android/net/ConnectivityManager } \\
\hline AP101 & getActiveNetworkInfo \\
\hline AP102 & getAllNetworkInfo \\
\hline AP103 & getMobileDataEnabled \\
\hline AP104 & getNetworkInfo \\
\hline AP105 & requestRouteToHost \\
\hline AP106 & setMobileDataEnabled \\
\hline AP107 & startUsingNetworkFeature \\
\hline AP108 & stopUsingNetworkFeature \\
\hline \multicolumn{2}{|c|}{ AP109 : path: android/net/ } \\
\hline AP109 & NetworkInfo;->isConnectedOrConnecting \\
\hline \multicolumn{2}{|c|}{ AP110-AP127 :path: android/net/wifi/WifiManager } \\
\hline AP110 & \$WifiLock;->acquire \\
\hline AP111 & $\$$ WifiLock;->release \\
\hline AP112 & addNetwork \\
\hline AP113 & disableNetwork \\
\hline AP114 & disconnect \\
\hline AP115 & enableNetwork \\
\hline AP116 & getConfiguredNetworks \\
\hline AP117 & getConnectionInfo \\
\hline AP118 & getDhcpInfo \\
\hline AP119 & getScanResults \\
\hline AP120 & getWifiState \\
\hline AP121 & isWifiEnabled \\
\hline AP122 & reconnect \\
\hline AP123 & removeNetwork \\
\hline AP124 & saveConfiguration \\
\hline AP125 & setNumAllowedChannels \\
\hline AP126 & setWifiEnabled \\
\hline
\end{tabular}




\begin{tabular}{|c|c|}
\hline AP127 & startScan \\
\hline \multicolumn{2}{|c|}{ AP128-AP130 : path: android/os/PowerManager } \\
\hline AP128 & \$WakeLock;->acquire \\
\hline AP129 & \$WakeLock;->release \\
\hline AP130 & reboot \\
\hline \multicolumn{2}{|c|}{ AP131-AP132 : path: android/os/Vibrator } \\
\hline AP131 & cancel \\
\hline AP132 & vibrate \\
\hline \multicolumn{2}{|c|}{ AP133-AP149 : path: android/provider } \\
\hline AP133 & Browser;->clearHistory \\
\hline AP134 & Browser;->clearSearches \\
\hline AP135 & Browser;->getAllBookmarks \\
\hline AP136 & Browser;-> getAllVisitedUrls \\
\hline AP137 & Contacts\$People;->addToMyContactsGroup \\
\hline AP138 & Contacts\$People;->createPersonInMyContactsGroup \\
\hline AP139 & Contacts\$People;->setPhotoData \\
\hline AP140 & ContactsContract\$Contacts;->getLookupUri \\
\hline AP141 & ContactsContract\$Contacts;->openContactPhotoInputStream \\
\hline AP142 & Settings\$Secure;->putInt \\
\hline AP143 & Settings\$Secure;->putLong \\
\hline AP144 & Settings $\$$ Secure;->putString \\
\hline AP145 & Settings\$System;->putInt \\
\hline AP146 & Settings\$System;->putString \\
\hline AP147 & Telephony\$Sms\$Sent;->addMessage \\
\hline AP148 & Telephony\$Sms;->addMessageToUri \\
\hline AP149 & Telephony\$Threads;-> getOrCreateThreadId \\
\hline \multicolumn{2}{|c|}{ AP150: path: android/speech/SpeechRecognizer } \\
\hline AP150 & startListening \\
\hline \multicolumn{2}{|c|}{ AP151-AP170 : path: android/telephony } \\
\hline AP151 & gsm/SmsManager;->sendMultipartTextMessage \\
\hline AP152 & gsm/SmsManager;->sendTextMessage \\
\hline AP153 & PhoneNumberUtils;->isVoiceMailNumber \\
\hline AP154 & SmsManager;->copyMessageToIcc \\
\hline AP155 & SmsManager;->deleteMessageFromIcc \\
\hline AP156 & SmsManager;-> getAllMessagesFromIcc \\
\hline AP157 & SmsManager;->sendDataMessage \\
\hline AP158 & SmsManager;->sendMultipartTextMessage \\
\hline AP159 & SmsManager;->sendTextMessage \\
\hline AP160 & SmsManager;-> updateMessageOnIcc \\
\hline AP161 & TelephonyManager;->getCellLocation \\
\hline AP162 & TelephonyManager;->getDeviceId \\
\hline AP163 & TelephonyManager;->getDeviceSoftwareVersion \\
\hline AP164 & TelephonyManager;->getLine1Number \\
\hline
\end{tabular}




\begin{tabular}{|c|c|}
\hline AP165 & TelephonyManager;-> getNeighboringCellInfo \\
\hline AP166 & TelephonyManager;->getSimSerialNumber \\
\hline AP167 & TelephonyManager;->getSubscriberId \\
\hline AP168 & TelephonyManager;->getVoiceMailAlphaTag \\
\hline AP169 & TelephonyManager;->getVoiceMailNumber \\
\hline AP170 & TelephonyManager;->listen \\
\hline \multicolumn{2}{|c|}{ AP171-AP1702 : path: com/android/internal/telephony/CallerInf } \\
\hline AP171 & getCallerInfo \\
\hline AP172 & markAsVoiceMail \\
\hline \multicolumn{2}{|c|}{ AP173- AP180 : path: java } \\
\hline AP173 & lang/Runtime;->exec \\
\hline AP174 & net/HttpURLConnection;->connect \\
\hline AP175 & net/ServerSocket;->bind \\
\hline AP176 & net/URL;->getContent \\
\hline AP177 & net/URL;->openConnection \\
\hline AP178 & net/URL;->openStream \\
\hline AP179 & net/URLConnection;->connect \\
\hline AP180 & net/URLConnection;->getInputStream \\
\hline \multicolumn{2}{|c|}{ AP181 : path: org/apache } \\
\hline AP181 & http/impl/client/DefaultHttpClient;->execute \\
\hline \multicolumn{2}{|c|}{ AP182-AP184 : path: Cipher } \\
\hline AP182 & AES \\
\hline AP183 & AES/CBC/PKCS5Padding \\
\hline AP184 & RSA/ECB/PKCS1Padding \\
\hline AP185 & Crypto-Cipher \\
\hline AP186 & Get-Package-Info \\
\hline AP187 & Get-System-Service \\
\hline AP188 & Http-Post \\
\hline AP189 & Obfuscation-base64 \\
\hline AP190 & Send-SMS \\
\hline
\end{tabular}

Table 4. 14 APIs extracted from training dataset that are related with sms and camera

\begin{tabular}{|c|c|c|}
\hline Nominal Data & $\begin{array}{c}\text { API String } \\
\text { (starts with : android) }\end{array}$ & Function \\
\hline AP101 & $\begin{array}{l}\text { net/ConnectivityManager;- } \\
\text { > getActiveNetworkInfo }\end{array}$ & $\begin{array}{l}\text { It returns details about the currently active } \\
\text { default data network }\end{array}$ \\
\hline AP102 & $\begin{array}{l}\text { net/ConnectivityManager;- } \\
>\text { getAllNetworkInfo }\end{array}$ & $\begin{array}{l}\text { It returns connection status information } \\
\text { about all network types supported by the } \\
\text { device }\end{array}$ \\
\hline AP104 & net/ConnectivityManager;->getNetworkInfo & $\begin{array}{l}\text { It returns connection status information } \\
\text { about a particular network type. }\end{array}$ \\
\hline AP147 & provider/Telephony\$Sms\$Sent;->addMessage & $\begin{array}{l}\text { It contains all sent text-based SMS mes- } \\
\text { sages in the SMS app. }\end{array}$ \\
\hline AP151 & $\begin{array}{l}\text { telephony/gsm/SmsManager;- } \\
\text { >sendMultipartTextMessage }\end{array}$ & It sends a multi-part text based SMS. \\
\hline
\end{tabular}




\begin{tabular}{|c|c|c|}
\hline AP152 & $\begin{array}{l}\text { telephony/gsm/SmsManager;- } \\
\text { >sendTextMessage }\end{array}$ & It sends a text based SMS. \\
\hline AP157 & telephony/SmsManager;->sendDataMessage & $\begin{array}{l}\text { It sends a data based SMS to a specific } \\
\text { application port. }\end{array}$ \\
\hline AP158 & $\begin{array}{l}\text { telephony/SmsManager;- } \\
\text { >sendMultipartTextMessage }\end{array}$ & It sends a multi-part text based SMS. \\
\hline AP159 & telephony/SmsManager;->sendTextMessage & It sends a text based SMS \\
\hline AP78 & hardware/Camera; ->open & $\begin{array}{l}\text { It creates a new Camera object to access } \\
\text { the first back-facing camera on the device. }\end{array}$ \\
\hline AP77 & $\begin{array}{l}\text { content/pm/PackageManager;- } \\
>\text { setComponentEnabledSetting }\end{array}$ & It enable application in package manager. \\
\hline AP82 & $\begin{array}{l}\text { location/LocationManager;- } \\
\text { >getLastKnownLocation }\end{array}$ & $\begin{array}{l}\text { It returns a location indicating the data } \\
\text { from the last known location fix obtained } \\
\text { from the given provider. }\end{array}$ \\
\hline AP 86 & $\begin{array}{l}\text { location/LocationManager;- } \\
\text { >requestLocationUpdates }\end{array}$ & $\begin{array}{l}\text { It registers for location updates using the } \\
\text { named provider. }\end{array}$ \\
\hline AP107 & $\begin{array}{l}\text { net/ConnectivityManager;- } \\
>\text { startUsingNetworkFeature }\end{array}$ & It starts network feature in an application. \\
\hline
\end{tabular}

As for Table V, it displays 5 extracted APIs from the social media apps that matched and could be associated with SMS and camera exploitation from the training dataset. While Table VI displays, 138 permissions extracted from the training dataset. Table VII presents 15 permissions that are related with SMS and camera. Table VIII presents 4 permissions extracted from the social apps that matched and could be associated with SMS and camera exploitation from the training dataset.

Table 5. API associated with sms and camera from social media app

\begin{tabular}{|c|c|}
\hline Social Media Apps & API \\
\hline SM1 & AP78, AP177 \\
\hline SM2 & AP78, AP82 \\
\hline SM3 & AP78, AP107 \\
\hline SM4 & AP78 \\
\hline SM5 & AP78, AP86 \\
\hline
\end{tabular}

Table 6. Permission extracted from training dataset

\begin{tabular}{|l|l|}
\hline \multicolumn{1}{|c|}{ Nominal data } & \multicolumn{1}{|c|}{ Permission } \\
\hline Q1-Q7: path: Access & \\
\hline Q1 & Checkin_properties \\
\hline Q2 & Coarse_Location \\
\hline Q3 & Fine_Location \\
\hline Q4 & Location_Extra_Commands \\
\hline Q5 & Network_State \\
\hline
\end{tabular}




\begin{tabular}{|c|c|}
\hline Q6 & Notification_Policy \\
\hline Q7 & Wifi_State \\
\hline \multicolumn{2}{|c|}{ Q8: path: Account } \\
\hline Q8 & Manager \\
\hline \multicolumn{2}{|c|}{ Q9: path: Add } \\
\hline Q9 & Voicemail \\
\hline \multicolumn{2}{|c|}{ Q10: path: Battery } \\
\hline Q10 & Stats \\
\hline \multicolumn{2}{|c|}{ Q11-Q34: path: Bind } \\
\hline Q11 & Accessibility_Service \\
\hline Q12 & Appwidget \\
\hline Q13 & Carrier_Messaging_Service \\
\hline Q14 & Carrier_Services \\
\hline Q15 & Chooser_Target_Service \\
\hline Q16 & Condition_Provider_Service \\
\hline Q17 & Device_Admin \\
\hline Q18 & Dream_Service \\
\hline Q19 & Incall_Service \\
\hline Q20 & Input_Method \\
\hline Q21 & Midi_Device_Service \\
\hline Q22 & Nfc_Service \\
\hline Q23 & Notification_Listener_Service \\
\hline Q24 & Print_Service \\
\hline Q25 & Quick_Settings_Tile \\
\hline Q26 & Remoteviews \\
\hline Q27 & Screening_Service \\
\hline Q28 & Telecom_Connection_Service \\
\hline Q29 & Text_Service \\
\hline Q30 & Tv_Input \\
\hline Q31 & Voice_Interaction \\
\hline Q32 & Vpn_Service \\
\hline Q33 & Vr_Listener_Service \\
\hline Q34 & Wallpaper \\
\hline \multicolumn{2}{|c|}{ Q35-Q37: path: Bluetooth } \\
\hline Q35 & Same as path \\
\hline Q36 & Admin \\
\hline Q37 & Privileged \\
\hline \multicolumn{2}{|c|}{ Q38: path: Body_Sensors } \\
\hline Q38 & Same as path \\
\hline \multicolumn{2}{|c|}{ Q39-Q42: path: Broadcast } \\
\hline Q39 & Package_Removed \\
\hline Q40 & Sms \\
\hline Q41 & Sticky \\
\hline
\end{tabular}




\begin{tabular}{|c|c|}
\hline Q42 & Wap_Push \\
\hline \multicolumn{2}{|c|}{ Q43-Q44: path: Call } \\
\hline Q43 & Phone \\
\hline Q44 & Privileged \\
\hline \multicolumn{2}{|c|}{ Q45: path: Camera } \\
\hline Q45 & Same as path \\
\hline \multicolumn{2}{|c|}{ Q46-Q48: path: Capture } \\
\hline Q46 & Audio_Output \\
\hline Q47 & Secure_Video_Output \\
\hline Q48 & Video_Output \\
\hline \multicolumn{2}{|c|}{ Q49-Q53: path: Change } \\
\hline Q49 & Component_Enabled_State \\
\hline Q50 & Configuration \\
\hline Q51 & Network_State \\
\hline Q52 & Wifi_Multicast_State \\
\hline Q53 & Wifi_State \\
\hline \multicolumn{2}{|c|}{ Q54: path: Clear_App } \\
\hline Q54 & Cache \\
\hline \multicolumn{2}{|c|}{ Q55: path: Control_Location } \\
\hline Q55 & Updates \\
\hline \multicolumn{2}{|c|}{ Q55-Q57: path: Delete } \\
\hline Q56 & Cache_Files \\
\hline Q57 & Packages \\
\hline \multicolumn{2}{|c|}{ Q58: path: Diagnostic } \\
\hline \multicolumn{2}{|c|}{ Q59: path: Disable_Keyguard } \\
\hline \multicolumn{2}{|c|}{ Q60: path: Dump } \\
\hline \multicolumn{2}{|c|}{ Q61: path: Expand_Status_Bar } \\
\hline \multicolumn{2}{|c|}{ Q62: path: Factory_Test } \\
\hline \multicolumn{2}{|c|}{ Q63-Q66: path: Factory_Test } \\
\hline Q63 & Accounts \\
\hline Q64 & Accounts_Privileged \\
\hline Q65 & Package_Size \\
\hline Q66 & Tasks \\
\hline \multicolumn{2}{|c|}{ Q67: path: Global_Search } \\
\hline \multicolumn{2}{|c|}{ Q68-Q70: path: Install } \\
\hline Q68 & Location_Provider \\
\hline Q69 & Packages \\
\hline Q70 & Shortcut \\
\hline \multicolumn{2}{|c|}{ Q71: path: Internet } \\
\hline \multicolumn{2}{|c|}{ Q72: path: Kill_Background_Processes } \\
\hline \multicolumn{2}{|c|}{ Q73:path: Location_Hardware } \\
\hline \multicolumn{2}{|c|}{ Q74:path: Manage_Documents } \\
\hline \multicolumn{2}{|c|}{ Q75: path: Master_Clear } \\
\hline
\end{tabular}




\begin{tabular}{|c|c|}
\hline Q76: $p$ & t_Control \\
\hline$Q 77-Q$ & \\
\hline Q77 & Audio_Settings \\
\hline Q78 & Phone_State \\
\hline Q79-Q & \\
\hline Q79 & Format_Filesystems \\
\hline Q80 & Unmount_Filesystems \\
\hline Q81: $p$ & \\
\hline Q82: $p$ & e_Stats \\
\hline Q83: $p$ & ivity \\
\hline Q84:p & oing_Calls \\
\hline Q85-Q & \\
\hline Q85 & Calendar \\
\hline Q86 & Call_Log \\
\hline Q87 & Contacts \\
\hline Q88 & External_Storage \\
\hline Q89 & Frame_Buffer \\
\hline Q90 & Input_State \\
\hline Q91 & Logs \\
\hline Q92 & Phone_State \\
\hline Q93 & Sms \\
\hline Q94 & Sync_Settings \\
\hline Q95 & Sync_Stats \\
\hline Q96 & Voicemail \\
\hline Q97:p & \\
\hline Q98- & \\
\hline Q98 & Boot_Completed \\
\hline Q99 & $\mathrm{Mms}$ \\
\hline Q100 & Sms \\
\hline Q101 & Wap_Push \\
\hline Q102: & \\
\hline Q103: & \\
\hline Q104- & \\
\hline Q104 & Ignore_Battery_Optimizations \\
\hline Q105 & Install_Packages \\
\hline Q106: & ages \\
\hline Q107: & id_Via_Message \\
\hline Q108: & \\
\hline Q109- & \\
\hline Q109 & Alarm \\
\hline Q110 & Always_Finish \\
\hline Q111 & Animation_Scale \\
\hline Q112 & Debug_App \\
\hline
\end{tabular}




\begin{tabular}{|c|c|}
\hline Q113 & Preferred_Applications \\
\hline Q114 & Process_Limit \\
\hline Q115 & Time \\
\hline Q116 & Time_Zone \\
\hline Q117 & Wallpaper \\
\hline Q118 & Wallpaper_Hints \\
\hline \multicolumn{2}{|c|}{ Q119: path: Signal_Persistent_Processes } \\
\hline \multicolumn{2}{|c|}{ Q120: path: Status_Bar } \\
\hline \multicolumn{2}{|c|}{ Q121: path:System_Alert_Window } \\
\hline \multicolumn{2}{|c|}{ Q122: path: Transmit_Ir } \\
\hline \multicolumn{2}{|c|}{ Q123: path: Uninstall_Shortcut } \\
\hline \multicolumn{2}{|c|}{ Q124: path: Update_Device_Stats } \\
\hline \multicolumn{2}{|c|}{ Q125: path: Use_Fingerprint } \\
\hline \multicolumn{2}{|c|}{ Q126: path: Use_Sip } \\
\hline \multicolumn{2}{|c|}{ Q127: path: Vibrate } \\
\hline \multicolumn{2}{|c|}{ Q128: path: Wake_Lock } \\
\hline \multicolumn{2}{|c|}{ Q129-Q138: path: Write } \\
\hline Q129 & Apn_Settings \\
\hline Q130 & Calendar \\
\hline Q131 & Call_Log \\
\hline Q132 & Contacts \\
\hline Q133 & External_Storage \\
\hline Q134 & Gservices \\
\hline Q135 & Secure_Settings \\
\hline Q136 & Settings \\
\hline Q137 & Sync_Settings \\
\hline Q138 & Voicemail \\
\hline
\end{tabular}

Table 7. 15 Permissions extracted from training dataset that are related with sms and camera

\begin{tabular}{|c|c|}
\hline $\begin{array}{l}\text { Nominal } \\
\text { Data }\end{array}$ & $\begin{array}{c}\text { Function } \\
\text { (To allow access for the following function) }\end{array}$ \\
\hline Q5 & Network information. \\
\hline Q14 & Binds with services in carrier apps \\
\hline Q40 & Broadcast SMS notification. \\
\hline Q87 & Reads user's contact information \\
\hline Q88 & Reads external storage. \\
\hline Q92 & $\begin{array}{l}\text { Reads to phone state (phone number, network information, any ongoing call status and } \\
\text { registered phone account }\end{array}$ \\
\hline Q93 & Reads SMS. \\
\hline Q99 & Monitors incoming MMS. \\
\hline Q100 & Receives SMS. \\
\hline Q101 & Receives WAP. \\
\hline Q108 & Sends SMS. \\
\hline
\end{tabular}




\begin{tabular}{|c|l|}
\hline Q132 & Writes user's contact information. \\
\hline Q133 & Writes external storage. \\
\hline Q45 & Accesses to the camera. \\
\hline Q88 & Reads from external storage. \\
\hline Q133 & Writes to external storage. \\
\hline Q48 & Captures video recording. \\
\hline
\end{tabular}

Table 8. Permission associated with sms and camera from social media apps

\begin{tabular}{|c|c|}
\hline Social Media Apps & Permission \\
\hline SM1 & Q40, Q45, Q48 \\
\hline SM2 & Q40, Q45, Q48 \\
\hline SM3 & Q40, Q45, Q48, Q108 \\
\hline SM4 & Q40, Q45, Q48 \\
\hline SM5 & Q40, Q45, Q48 \\
\hline
\end{tabular}

The significant of having 328 APIs and permissions (combination of Table III and VI) from the mobile botnets training dataset is, it could be used as guidance for the mobile apps developer on how the attackers could exploit the smartphone via API and permission. Furthermore, from the analysis, 29 APIs and permissions (from Table IV and Table VII) are related with SMS and camera. This represents $8.8 \%$ from the training dataset and could be used for SMS and camera exploitation. From 5 selected of social media apps, only total of 9 permissions and APIs that matched with the extracted APIs and permissions from Table IV and Table VII. This represents $2.7 \%$ from the training dataset. These APIs and permissions of SMS and camera might pose privacy and financial risks for smartphone users.

\section{Conclusion}

Based on the experiment conducted, it showed that social media apps could be used as the attacker's target for SMS and camera exploitation. Since Android-based application is in open-source form, malware may camouflage itself as a legitimate mobile application. The significant finding of this paper is the identification of normal API and permission for SMS and camera and possible of API and permission SMS and API exploitation. This extracted classification can be used as input or database for the development of mobile application for detection of social media exploitation.

\section{$5 \quad$ Acknowledgment}

The authors would like to express their gratitude to Universiti Sains Islam Malaysia (USIM) for the support and facilities provided. This research paper is funded under grant: [PPP/USG-0216/FST/30/15116] 


\section{$6 \quad$ References}

[1] Verma, S. Arora and P.Verma, "Android OS, its security and features", International Journal of Recent Research Aspects, Vol. 4, Issue 3, Sept 2017, pp. 241- 251.

[2] L. Thomson, "Tech firms take down WireX Android botnet", 2017, The Register, URL: https://www.theregister.co.uk/2017/08/28/tech firms take down wirex android botnet/

[3] P. R. K. Varma, K. P. Raj and K. V. S. Raju, "Android mobile security by detecting and classification of malware based on permissions using machine learning algorithms," 2017 International Conference on I-SMAC (IoT in Social, Mobile, Analytics and Cloud) (ISMAC), Palladam, 2017, pp. 294-299. https://doi.org/10.1109/i-smac.2017.8058358

[4] Talha, K. A., Alper, D. I., and Aydin, C., "APK Auditor: Permission-based Android malware detection system", Digital Investigation, 13, 2015, pp.1-14. https://doi.org/10.1016/ j.diin.2015.01.001

[5] Saracino, A., Sgandurra, D., Dini, G., and Martinelli, F., "MADAM: Effective and Efficient Behavior-based Android Malware Detection and Prevention", IEEE Transactions on Dependable and Secure Computing, 5971(c), 2016, pp. 1-1. https://oi.org/10.1109/ TDSC.2016.2536605

[6] Yerima, S. Y., Sezer, S., and Muttik, I., "High accuracy android malware detection using ensemble learning". IET Information Security, 9(6), 2015, pp. 313-320. https://doi.org/10.1049/iet-ifs.2014.0099

[7] EMB. Karbab, M. Debbabi, A. Derhab and D. Mouheb, "MalDozer: Automatic framework for android malware detection using deep learning", Digital Investigation, Vol. 24, 2018, pp. S48-S59, https://doi.org/10.1016/j.diin.2018.01.007

[8] Wang, Z., Cai, J., Cheng, S., and Li, W., "DroidDeepLearner: Identifying Android malware using deep learning. 37th IEEE Sarnoff Symposium, Sarnoff 2016, 2017, pp. 160165. https://doi.org/10.1109/SARNOF.2016.7846747

[9] Kamesh, SPN. and Priya, S.,"Security enhancement of authenticated RFID generation”. International Journal of Applied Engineering Research, 9(22), 2014, pp. 5968-5974. https://doi.org/10.1002/sec

[10] Li, J., Sun, L., Yan, Q., Li, Z., Srisa-an, W., and Ye, H. “Android Malware Detection”, IEEE Transactions on Industrial Informatics, 2018, 3203(c). https://doi.org/10.1109/TII. $\underline{2017.2789219}$

[11] Li, D., Wang, Z., Li, L., Wang, Z., Wang, Y., \& Xue, Y. (2017). FgDetector : Fine-grained Android Malware Detection, 311-318. https://doi.org/10.1109/DSC.2017.13

[12] Madihah Mohd Saudi and Muhammad "Afif b. Husainiamer, "Mobile Malware Classification via System Calls and Permission for GPS Exploitation" International Journal of Advanced Computer Science and Applications(IJACSA), 8(6), 2017. http://dx.doi.org/10.14569/IJACSA.2017.080636

[13] Burnap, P., French, R., Turner, F., \& Jones, K. (2018). Malware classification using self organising feature maps and machine activity data. Computers and Security, 73, 399-410. https://doi.org/10.1016/i.cose.2017.11.016

[14] Chen, S., Xue, M., Fan, L., Hao, S., Xu, L., Zhu, H., \& Li, B. (2018). Automated poisoning attacks and defenses in malware detection systems: An adversarial machine learning approach. Computers and Security, 73, 326-344. https://doi.org/10.1016/j.cose. $\underline{2017.11 .007}$

[15] Li, J., Sun, L., Yan, Q., Li, Z., Srisa-an, W., \& Ye, H. (2018). Android Malware Detection. IEEE Transactions on Industrial Informatics, 3203(c). https://doi.org/10.1109/TII.2017. 2789219

[16] Yerima, S. Y., \& Sezer, S. (2018). DroidFusion: A Novel Multilevel Classifier Fusion Approach for Android Malware Detection. IEEE Transactions on Cybernetics, 1-14. https://doi.org/10.1109/TCYB.2017.2777960 
[17] Wang, S., Yan, Q., Chen, Z., Yang, B., Zhao, C., \& Conti, M. (2018). Detecting Android Malware Leveraging Text Semantics of Network Flows. IEEE Transactions on Information Forensics and Security, 13(5), 1096-1109. https://doi.org/10.1109/TIFS.2017. 2771228

[18] E. Biglar Beigi, H. Hadian Jazi, N. Stakhanova and A. A. Ghorbani, "Towards effective feature selection in machine learning-based botnet detection approaches," 2014 IEEE Conference on Communications and Network Security, San Francisco, CA, 2014, pp.247255. https://doi.org/10.1109/cns.2014.6997492

\section{$7 \quad$ Authors}

Mohd Adib Azhar is a student at Faculty of Science and Technology (FST), Universiti Sains Islam Malaysia(USIM). His interest are in malwares and computer security

Madihah Mohd Saudi is an Associate Professor at Information Security and Assurance (ISA) Programme, Faculty of Science and Technology (FST), Universiti Sains Islam Malaysia (USIM), Malaysia. Currently she is the Chief Information Officer (CIO) at USIM and leading a group called as Cybersecurity and Systems Research Unit at Islamic Science Institute (ISI), USIM. She is also senior member for IEEE Computer Society and International Association of Computer Science and IT (IACSIT) and technical committee member on IEEE Security and Privacy and Pattern Analysis and Machine Intelligence. She has over 18 years of research experience in the fields of cyber security and data mining. She has published numerous of books, policies and academic papers in international journals related with computer security and data mining. She has also won a few international innovation awards related with cyber security. Her research interests are in cyber security, data mining and bioinspired computing. She holds 2 professional CERT related with Cyber Security from United States and received her PhD from University of Bradford, United Kingdom in Computer Security, MSc (Software Engineering) from University of Malaya (UM), and BSc(Hons) in Computer Science from National University of Malaysia (UKM) (madihah@usim.edu.my).

Dr. Azuan Ahmad is an academician at the Faculty of Science and Technology (FST), Universiti Sains Islam Malaysia (USIM). He obtained his Ph.D and M.Sc. in Computer Science from University Teknologi Malaysia. He received his B.Sc Honours in Computer Science (Information Security Assurance) from Universiti Sains Islam Malaysia. He is the certified professional in various area including cybersecurity, computer network and various operating systems. Until today, he is actively doing research in the area of cloud security, Internet of Things (IoT), Big Data and malware research (azuan@usim.edu.my).

Azreena Abu Bakar is an a lecturer at Faculty of Science and Technology (FST), Universiti Sains Islam Malaysia(USIM). Her interest are in computer security, knowledge management and information systems (azreena@usim.edu.my).

Article submitted 2019-01-15. Resubmitted 2019-02-28. Final acceptance 2019-03-15. Final version published as submitted by the authors. 